\title{
Evidence for differential expression of Notch receptors and their ligands in melanocytic nevi and cutaneous malignant melanoma
}

\author{
Daniela Massi ${ }^{1}$, Francesca Tarantini $^{2}$, Alessandro Franchi ${ }^{1}$, Milena Paglierani ${ }^{1}$, \\ Claudia Di Serio ${ }^{2}$, Silvia Pellerito ${ }^{2}$, Giuseppe Leoncini ${ }^{1}$, Giuseppe Cirino ${ }^{3}$, \\ Pierangelo Geppetti ${ }^{2}$ and Marco Santucci ${ }^{1}$ \\ ${ }^{1}$ Department of Human Pathology and Oncology, University of Florence, Florence, Italy; ${ }^{2}$ Department of \\ Critical Care Medicine and Surgery, University of Florence, Florence, Italy and ${ }^{3}$ Department of Experimental \\ Pharmacology, University of Naples, Naples, Italy
}

\begin{abstract}
The Notch signaling has been implicated in the regulation of self-renewal of adult stem cells and differentiation of precursors along a specific cell lineage, in normal embryonic development and organogenesis. There is also evidence that signaling through Notch receptors regulate cell proliferation and cell survival in several types of cancer, with opposing results depending on tissue context. No data are available in the literature concerning modulation of the expression of Notch receptors, and their ligands, in human cutaneous malignant melanoma. Here, we have investigated, for the first time, the expression of Notch-1, Notch-2, Jagged-1, Jagged-2 and Deltalike 1 proteins, by immunohistochemistry, in a series of benign and malignant human melanocytic lesions: five common melanocytic nevi, five 'dysplastic nevi' and 20 melanomas (five in situ, five T1-T2, five T3-T4 and five metastatic melanomas). We found that the expression of Notch-1 and Notch-2, as well as Notch ligands, was upregulated in 'dysplastic nevi' and melanomas as compared with common melanocytic nevi. These results indicate that the activation of Notch may represent an early event in melanocytic tumor growth and upregulation of Notch signaling may sustain tumor progression.
\end{abstract}

Modern Pathology (2006) 19, 246-254. doi:10.1038/modpathol.3800526; published online 9 December 2005

Keywords: Notch-1; Notch-2; Jagged-1; Jagged-2; Delta-like 1; melanoma

In normal embryonic development and organogenesis, the Notch signaling pathway plays a pivotal role in the regulation of cell fate decisions, such as self-renewal of adult stem cells and differentiation of precursors along a specific cell lineage. Depending on the cellular and tissue context, the Notch signals act through the regulation of cell proliferation, migration, differentiation and apoptosis. ${ }^{1-3}$ The Notch gene encodes a cell surface receptor first identified in Drosophila where a mutated protein caused 'notches' in the fly wing. The Notch gene encodes a transmembrane receptor, which is expressed in embryonic and adult cells. Notch ligands-Delta and Serrate in Drosophila, LAG-2 and APX-1 in Caenorhabditis elegans and Delta and

Correspondence: Professor D Massi, MD, Department of Human Pathology and Oncology, University of Florence, Viale GB Morgagni 85, Florence I-50134, Italy.

E-mail: Daniela.massi@UNIFI.IT

Received 27 July 2005; revised 17 October 2005; accepted 18 October 2005; published online 9 December 2005
Jagged in vertebrates-are also transmembrane proteins: the ligand is expressed on an adjacent cell and activates Notch signaling through a direct cell-cell interaction. In mammals, four Notch genes, Notch-1, $-2,-3$ and -4 , have been described. ${ }^{4-6}$ Notch proteins are transmembrane receptors that are activated by six trasmembrane ligands that in vertebrates have been identified in two Serrate-like proteins, Jagged-1 and -2 , as well as four Delta-like proteins, Delta-like$1,-2,-3$ and $-4 .^{7,8}$ Upon ligands' binding, Notch receptors are activated by proteolytic cleavage of the single transmembrane domain releasing the Notch intracellular domain $\left(\right.$ Notch $\left.^{\text {ic }}\right)$ from the plasma membrane, which translocates to the nucleus, where it often associates with the DNA-binding transcription factor, CLS (acronym for CBF1, LAG-1 and $\mathrm{Su}(\mathrm{H})) .{ }^{9}$ This complex of the Notch ${ }^{\text {ic }}$ and the transcription factor CLS has been shown to transactivate the basic helix-loop-helix transcription factor Hes1 gene, and then affecting the regulation of downstream target genes. ${ }^{10}$

In addition to fetal and adult development, there is substantial evidence that the Notch signaling 
system promotes cell proliferation and cancer growth. Interestingly, Notch activation that causes cellular transformation has been demonstrated to occur not only due to its own genetic aberration but also to ligand stimulation. ${ }^{11,12}$ Indeed, a number of reports have demonstrated upregulated Notch-1 activity in a variety of human cancer tissues, including breast cancer, ${ }^{13}$ gliomas, ${ }^{14}$ hematopoietic malignancies such as Hodgkin's and anaplastic large-cell lymphomas, ${ }^{12}$ and cholangiocarcinoma. ${ }^{15}$ In addition, Jagged-1 was found significantly more expressed in metastatic prostate cancer as compared with localized prostate cancer or benign prostate tissue. ${ }^{16}$

However, recent data suggest that Notch can act as an oncogene or function as a tumor suppressor depending on the cell type. ${ }^{17}$ In the skin, Notch-1 has a role in coordinating differentiation of squamous epithelia and deletion of Notch-1 expression in mouse skin resulted in hyperproliferation of the basal epidermal layer, culminating in the development of skin tumors in $95 \%$ of mice. ${ }^{18,19}$ Moreover, in cervical cancer and small-cell lung cancer, activation of Notch-1 signaling has shown inhibitory functions on cell growth, ${ }^{19-23}$ resulting in tumor suppression. Therefore, the role of Notch signaling in tumorigenesis is highly context dependent, and still far to be completely elucidated.

No data are available in the literature concerning expression of Notch proteins and their ligands in human cutaneous malignant melanoma. Microarray studies have suggested that Notch-2 mRNA is overexpressed in melanoma cells compared to nevi and normal melanocytes, ${ }^{24,25}$ and Jagged-2 mRNA is strongly upregulated in highly invasive melanoma cell lines. ${ }^{26} \mathrm{~A}$ thorough investigation of the tissue and cellular distribution of Notch receptors and their ligands, during melanoma development, will contribute to the understanding of the role of Notchmediated intracellular signaling in tumor growth. In the current study, for the first time, the expression pattern of two Notch proteins, Notch-1 and Notch-2, and three ligands, Jagged-1, Jagged-2 and Delta-like 1 , was investigated, by immunohistochemistry, in human specimens ranging from benign to highly malignant melanocytic lesions.

\section{Materials and methods}

\section{Specimen Selection}

The study series included five cutaneous common melanocytic nevi, five 'dysplastic nevi', five cutaneous in situ melanomas, five pT1 and pT2 invasive melanomas, five pT3 and pT4 invasive melanomas and five subcutaneous melanoma metastases. All samples, with the exemption of subcutaneous melanoma metastases, included both tumor and normal adjacent epidermis. Histopathological criteria for the diagnosis of lesional categories were those currently employed. ${ }^{27}$ In particular, the major architectural and cytological features for diagnosis of 'dysplastic nevi' included central dermal nevic component with a peripheral extension of a junctional component (so-called 'shoulder' phenomenon'), nested and single melanocytes mainly near the tips and sides of elongated epidermal rete ridges, stromal reactions (including concentric eosinophilic and lamellar fibroplasia) and mild to moderate cytological atipia. ${ }^{27} \mathrm{~A}$ diagnosis of in situ melanoma was made in the presence of increased numbers of atypical melanocytes, both in solitary units and/or in nests at the dermoepidermal junction and at all levels of the epidermis, with possible extension of melanocytes far down adnexal structures. Cytologically, melanocytes show a marked variation of size and shape and display enlarged, pleomorphic nuclei. ${ }^{27}$

Patients' data, including age, sex, anatomic tumor site, disease-specific survival, and status, were retrospectively collected. The median age of patients with in situ melanoma was 43.3 years (range 31-57 years). Three patients were females, and two patients were males. Tumor site distribution was upper and lower extremities (four cases) and trunk (one case). The median age of patients with primary invasive melanomas was 51.2 years (range 30-76 years). Six patients were females and four patients were males. The tumor site distribution was as follows: upper and lower extremities (four cases) and trunk (one case). Tumor site distribution was as follows: head and neck, two patients; upper and lower extremities, five patients; trunk, two patients; and acral regions, one patient. In addition, histopathological slides were reviewed to retrieve the following data: tumor thickness, presence of ulceration, Clark's level, and histotype.

\section{Immunohistochemistry}

Sections of $4 \mu \mathrm{m}$ thickness were cut from tissue blocks of formalin-fixed, paraffin-embedded samples obtained from the Department of Human Pathology and Oncology, University of Florence. Slides were deparaffined in Bio-Clear (Bio-Optica, Milano, Italy) and hydrated with grade ethanol concentrations. Tissue sections were labeled with the following antibodies: goat anti-human Notch-1 (sc-6014), goat anti-human Notch-2 (sc-5545), goat anti-human Jagged-1 (sc-6011), goat anti-human Jagged-2 (sc-8157), and rabbit anti-human Deltalike 1 (sc-9107) (Santa Crutz Biotechnology Inc.) (Table 1). Briefly, antigen retrieval was performed by microwave pretreatment (Microwave MicroMED T/T Mega, Milestone, Bergamo, Italy) in EDTA $1 \mathrm{mM}$, $\mathrm{pH}$ 8.0, for $30 \mathrm{~min}$; endogenous peroxidase activity was blocked by immersing slides in distilled water, containing $3.0 \%$ hydrogen peroxide, for $20 \mathrm{~min}$. Nonspecific antigen sites were blocked with normal horse serum (UltraVision, LabVision, Fremont, CA, USA), then the sections were incubated with 
primary antibodies at the indicated dilutions (Table 1). Staining was achieved using appropriate biotinconjugated anti-goat secondary antibody (IgG, Biomedicals, Verona, Italy), biotinylated goat anti- polyvalent (Ultravision) and streptavidin-peroxidase (UltraVision). Bound antibodies were visualized using aminoethylcarbazol (LabVision) as chromogen. Nuclei were slightly counterstained

Table 1 List of antibodies used for immunohistochemistry

\begin{tabular}{|c|c|c|c|c|}
\hline Antibody & Source & Dilution & $\begin{array}{l}\text { Incubation } \\
\text { time (h) }\end{array}$ & Pretreatment \\
\hline Notch-1 (goat, polyclonal) & Santa Cruz & $1: 30$ & 1 & $\begin{array}{l}\text { Antigen retrieval in EDTA } 1 \mathrm{mM} \text {, } \mathrm{pH} 8.0 \text {, for } 30 \mathrm{~min} \text { in } \\
\text { microwave oven }\end{array}$ \\
\hline Notch-2 (goat, polyclonal) & Santa Cruz & $1: 30$ & 2 & $\begin{array}{l}\text { Antigen retrieval in EDTA } 1 \mathrm{mM} \text {, pH 8.0, for } 30 \mathrm{~min} \text { in } \\
\text { microwave oven }\end{array}$ \\
\hline Jagged-1 (goat, polyclonal) & Santa Cruz & $1: 30$ & 2 & $\begin{array}{l}\text { Antigen retrieval in EDTA } 1 \mathrm{mM} \text {, } \mathrm{pH} 8.0 \text {, for } 30 \mathrm{~min} \text { in } \\
\text { microwave oven }\end{array}$ \\
\hline Jagged-2 (goat, polyclonal) & Santa Cruz & $1: 30$ & 2 & $\begin{array}{l}\text { Antigen retrieval in EDTA } 1 \mathrm{mM} \text {, pH 8.0, for } 30 \mathrm{~min} \text { in } \\
\text { microwave oven }\end{array}$ \\
\hline Delta-like 1 (rabbit, polyclonal) & Santa Cruz & $1: 30$ & 2 & $\begin{array}{l}\text { Antigen retrieval in EDTA } 1 \mathrm{mM} \text {, } \mathrm{pH} \text { 8.0, for } 30 \mathrm{~min} \text { in } \\
\text { microwave oven }\end{array}$ \\
\hline
\end{tabular}
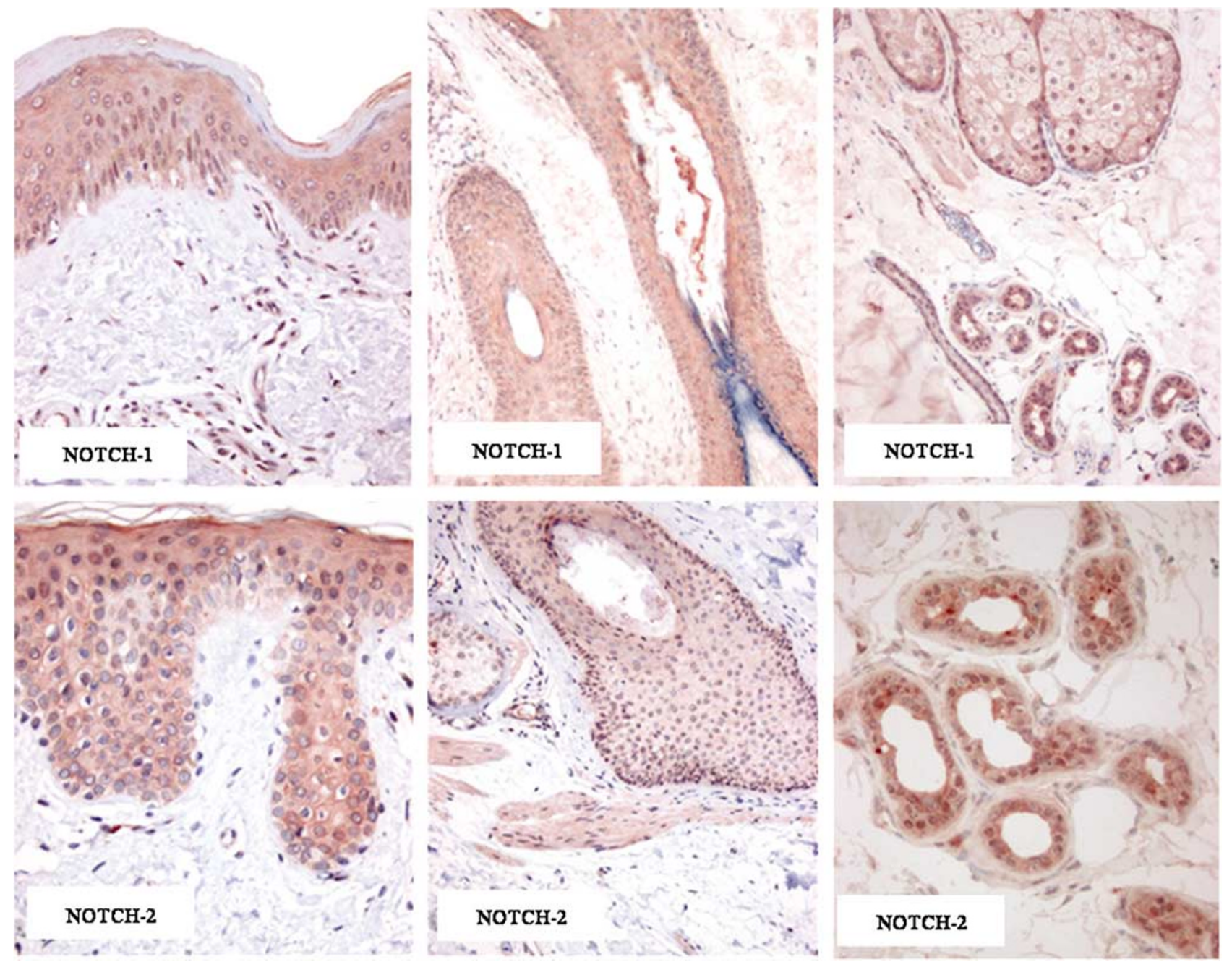

Figure 1 Notch-1 in normal skin is diffusely expressed in suprabasal epidermal keratinocytes, with only faint and focal staining in the granular layer and stratum corneum (upper left). Notch-1 expression is observed in epithelium of hair follicles (upper center), sweat glands, sebaceous glands, and blood vessels (upper right). Notch-2 strong immunoreactivity is seen in the basal and suprabasal spinous layers (lower left), hair follicles and sebaceous lands (lower center), as well as eccrine gland structures (lower right). 
with Mayer's hematoxylin. Negative controls were performed by substituting the primary antibody with a nonimmune serum. Control sections were treated in parallel with the samples, in the same run. Immunostained sections were independently assessed by three observers (DM, AF, and MS). The results were expressed according to semiquantitative criteria as follows: negative staining; low expression (1-20\% of positive cells); medium expression (21-60\% of positive cells); and high expression (more than $60 \%$ of positive cells). ${ }^{28}$ The staining intensity was scored on a scale as faint, moderate, or strong. Owing to the small number of samples, no statistical analysis of results was conducted.

\section{Results}

Notch-1 immunohistochemical expression was mostly confined to the cell cytoplasm, with minimal peripheral membrane and rare nuclear pattern. Notch-2 immunostaining was mostly observed in the cell cytoplasm, with focal peripheral membrane and occasional nuclear pattern. Interestingly, we noticed high levels of Notch-2 nuclear staining in specimens from melanoma metastases. Evaluation of Jagged-1, Jagged-2, and Delta-like 1 immunoreac- tions displayed predominantly cytoplasmic and plasma membrane staining in either normal or neoplastic cells, and no consistent nuclear pattern was observed.

\section{Immunohistochemical Study of Normal Adjacent Epidermis and Adnexal Structures}

In the skin, Notch-1 was strongly and diffusely expressed in suprabasal epidermal keratinocytes, with only faint and focal staining in the granular layer and stratum corneum (Figure 1). Interestingly, immunostaining of basal layer keratinocytes demonstrated that Notch-1 was barely detectable or absent in normal skin and in the epidermis overlying common melanocytic nevi, whereas enhanced expression was detected in keratinocytes overlying neoplastic cells. A high expression level of Notch-1 was consistently observed in the outer and inner root sheath of hair follicles, sweat glands, sebaceous glands; a positive immunostaining was also detected in dermal fibroblasts, scattered lymphocytes, and blood vessels. Notch-2 immunoreactivity was predominantly distributed to the basal and suprabasal spinous layers, and was absent to faint in the upper superficial layers. Scattered dermal fibroblasts, hair follicles, sebaceous, and eccrine gland structures

Table 2 Distribution of Notch-1, Notch-2, Jagged-1, Jagged-2, and Delta-like 1 expression in common nevi, atypical nevi, and malignant melanoma $(n=30)$

$$
C M N(\mathrm{n}=5) \quad D N(\mathrm{n}=5) \quad M I S(\mathrm{n}=5) \quad M M T 1-T 2(\mathrm{n}=5) \quad M M T 3-T 4(\mathrm{n}=5) \quad M M M e t(\mathrm{n}=5)
$$

\section{Notch-1}

Negative staining

Low expression

Medium expression

High expression

Notch-2

Negative staining

Low expression

Medium expression

High expression

Jagged-1

Negative staining

Low expression

Medium expression

High expression

Jagged-2

Negative staining

Low expression

Medium expression

High expression

Delta-like 1

Negative staining

Low expression

Medium expression

High expression

$\begin{array}{ll}4 & \\ - & \\ - & \\ & \\ - & \\ - & \end{array}$

$\frac{-}{5}$

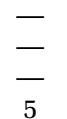

$\frac{-}{5}$

$\frac{-}{5}$

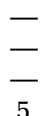

-
4

$-$

-
1
4

-

\begin{tabular}{l}
- \\
\hline 1 \\
4
\end{tabular}

$\begin{array}{ll}3 & - \\ - & -\end{array}$

$\frac{-}{1}$

-
1
4

-
1
4

-

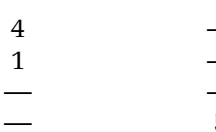

$\frac{7}{5}$

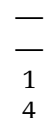

$\frac{-}{5}$

$\frac{7}{5}$

-
2

5
-
-

-

$\mathrm{NMC}=$ common melanocytic nevus; $\mathrm{DN}=$ 'dysplastic nevus'; $\mathrm{MM}=$ melanoma; MM Met = melanoma metastasis; low expression =1-20\% of positive cells; medium expression $=21-60 \%$ of positive cells; high expression $=$ more than $60 \%$ of positive cells. 
also stained positively (Figure 1). Jagged-1 and Jagged-2 were diffusely expressed in the cytoplasm and plasma membrane of basal and suprabasal keratinocytes, up to the granular cell layer, as well as in adnexal structures, although more weakly. Basal and suprabasal keratinocytes, as well as adnexal structures, also expressed low immunoreactivity for Delta-like 1 protein.

\section{Immunohistochemical Study of Cutaneous Melanocytic Lesions}

The results of the immunohistochemical investigations are summarized in Table 2. Normal epidermal melanocytes generally showed faint to moderate Notch-1 staining, although occasionally the immuno- labelling was difficult to evaluate, owing to the presence of a clear perinuclear halo, resulting from marked cytoplasmic retraction. A similar pattern was also seen for Notch-2 and for all three ligands examined.

In common melanocytic nevi, Notch-1, Notch-2, Jagged-1, and Jagged-2 staining was absent in the majority of melanocytes, with only a faint immunoreactivity in a small number of cells (low expression) (Figure 2). In particular, the junctional component of common melanocytic nevi was mostly negative, or negligible in a few cases (Figure 3, left side). Deltalike 1 staining was consistently absent. Most 'dysplastic nevi' displayed a moderate Notch-1 and Notch-2 expression in both the junctional and the intradermal component of the majority of melanocytes (high expression) (Figures 3 and 4), in a
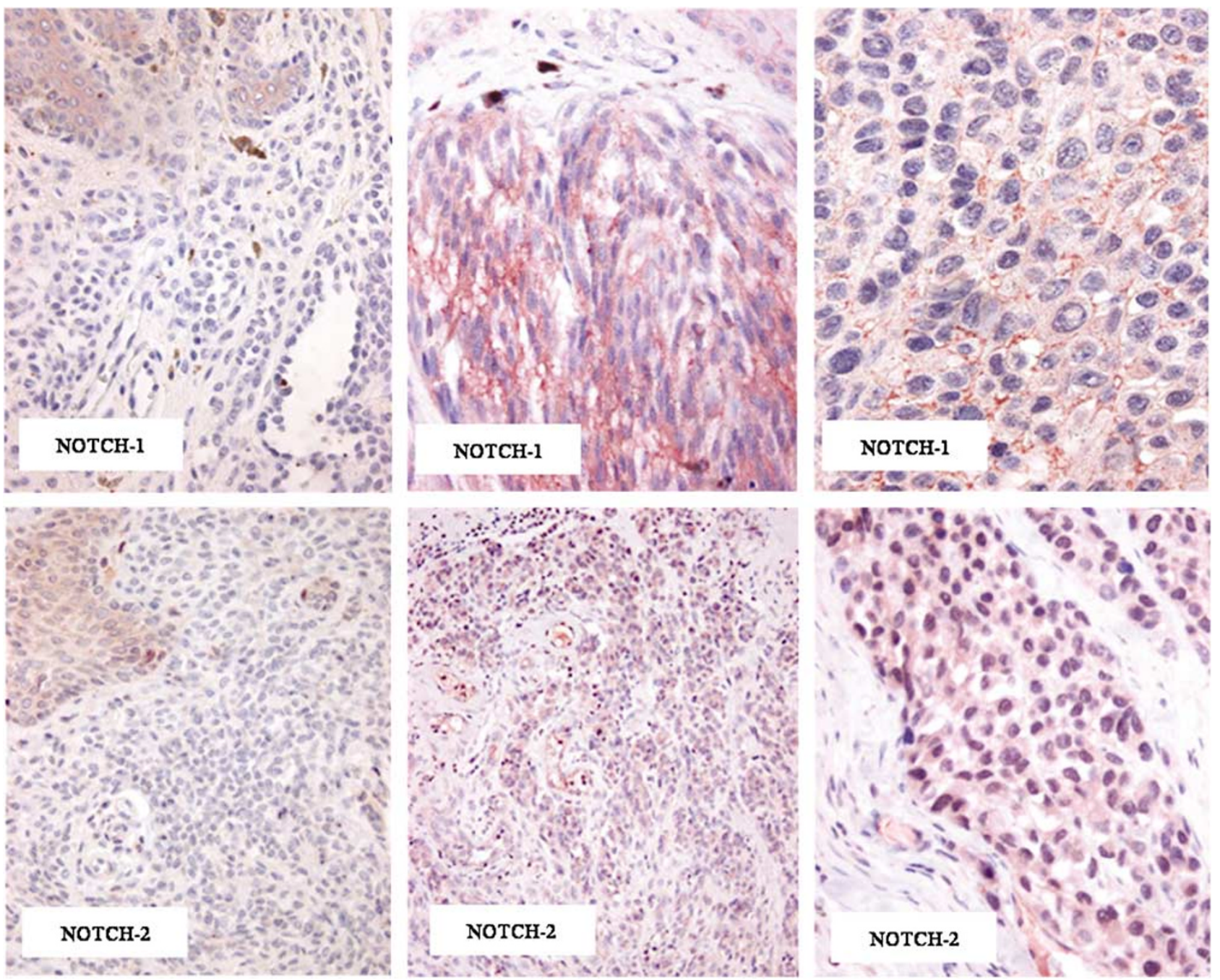

Figure 2 Notch-1 in common melanocytic nevus (upper left). Note the absence of Notch-1 immunostaining in nevus cells and basal keratinocytes, whereas the overlying suprabasal keratinocytes within the epidermis are positive. The invasive component of a thick melanoma shows an intense and diffuse Notch-1 cytoplasmic staining (upper center). The overlying epidermis is also positive. Subcutaneous melanoma metastasis displaying diffuse cytoplasmic and membrane Notch-1 expression (upper right). Notch-2 is negative in melanocytes of common melanocytic nevus (lower left). A thick melanoma showing diffuse Notch-2 staining. (lower center). Notch-2 cytoplasmic moderate positivity in subcutaneous metastasis (lower right). 

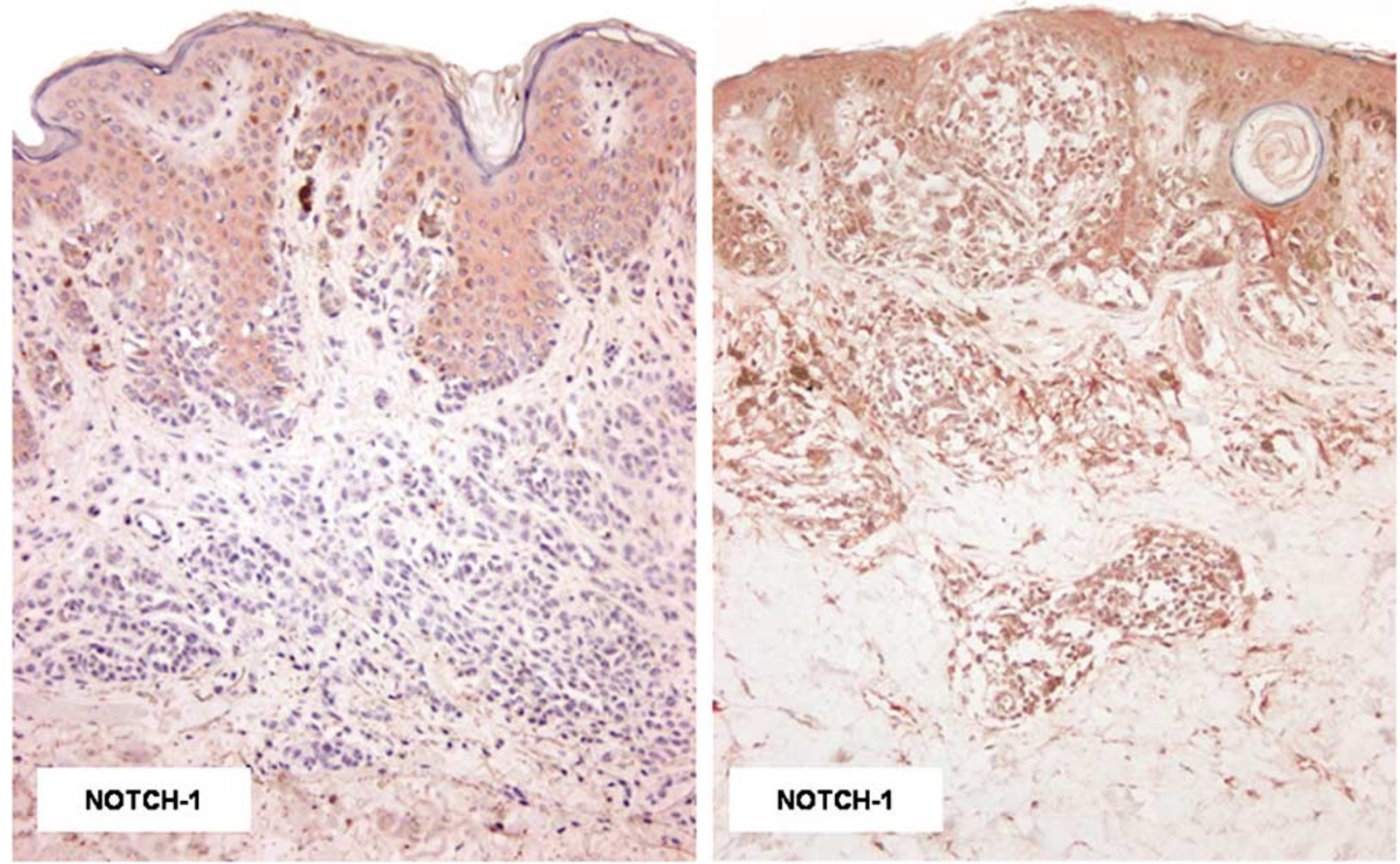

Figure 3 Notch-1 in common melanocytic nevus (left) and 'dysplastic nevus' (right). Note that the junctional component of the common nevus is negative, whereas the junctional component of the 'dysplastic nevus' is Notch-1 positive.

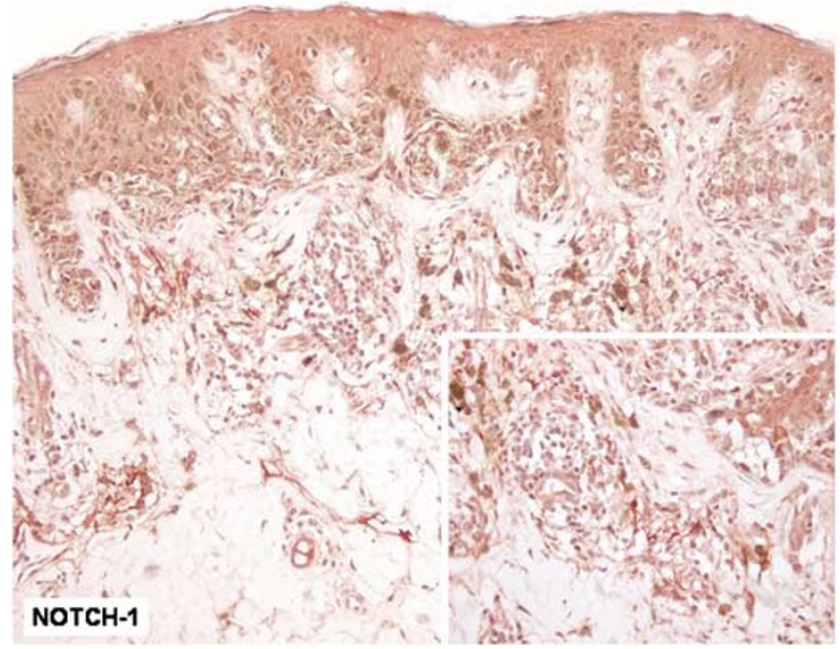

Figure 4 'Dysplastic nevus'. Notch-1 positivity is observed in the majority melanocytes mostly arranged in bridging nests at the dermoepidermal junction.

pattern similar to that displayed by in situ melanomas. Same results were obtained for Notch ligands.

Primary invasive melanomas, irrespective of tumor thickness, showed moderate to high levels of expression of Notch-1, Notch-2, and their ligands in the majority of tumor cells (high expression), with a stronger and more diffuse Jagged-2 staining (Figures 5 and 6 ). The immunoreactivity was comparable in

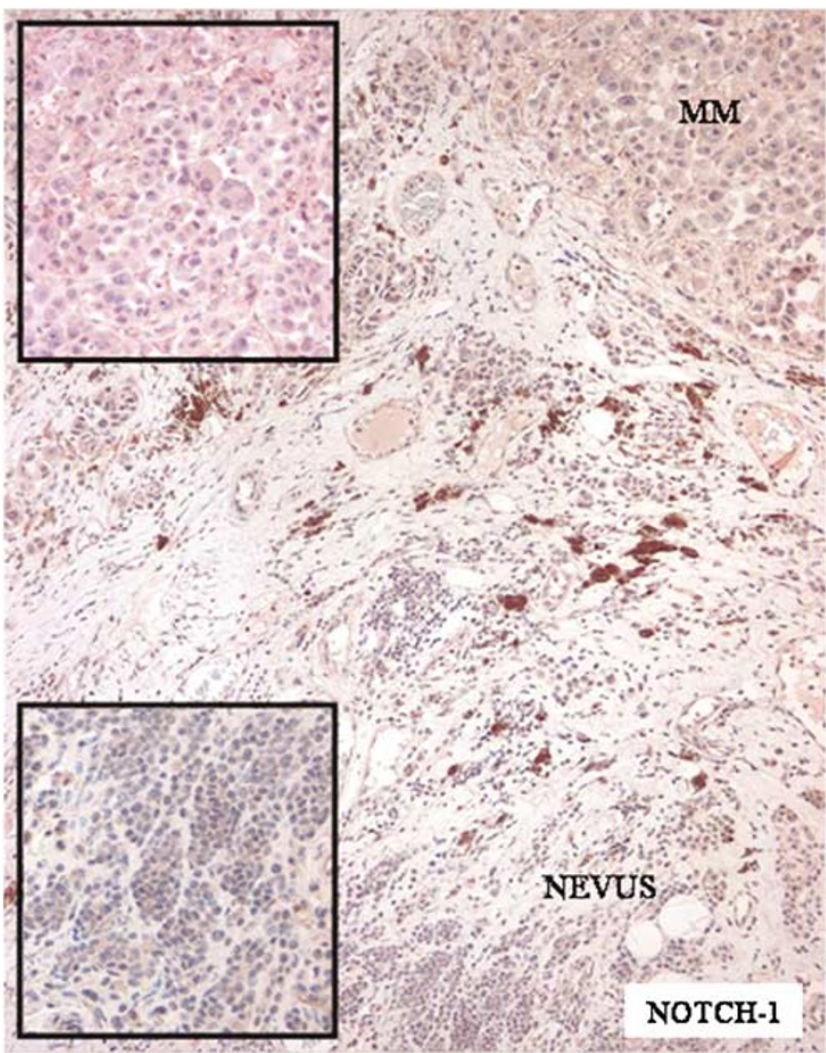

Figure 5 Nevus-associated melanoma. Melanoma cells (upper part) are markedly Notch-1 positive, whereas the adjacent nevus cells (lower part) show faint to absence of staining. 

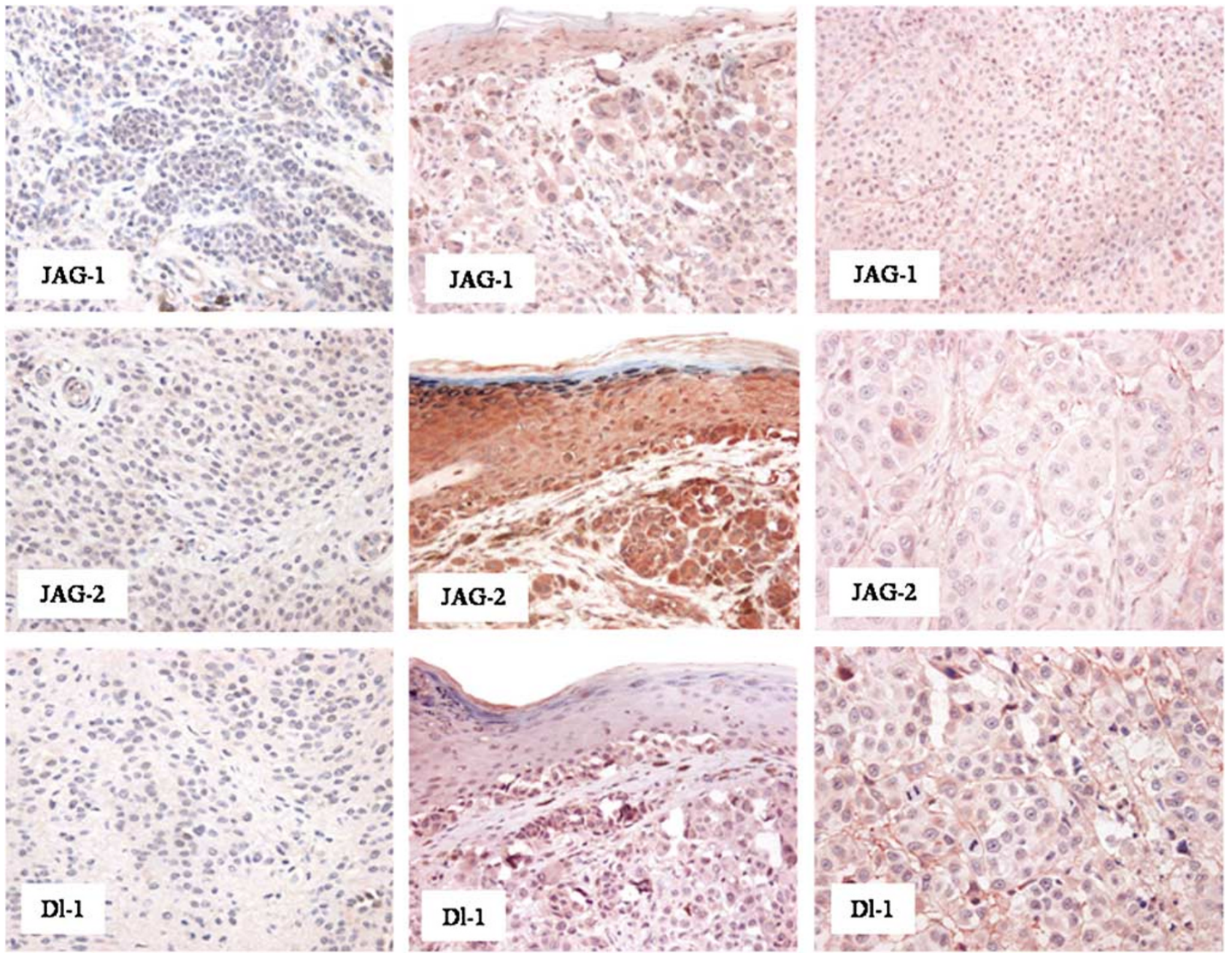

Figure 6 Immunohistochemical staining for Jagged-1, Jagged-2, and Delta-like-1 in common nevi (left), primary melanomas (center), and melanoma metastases (right). In common nevi, Jagged-1 and Jagged-2 show faint staining in a minority of cells, whereas Delta-like-1 is completely negative. Primary invasive melanomas show moderate Jagged-1 and Delta-like-1 expression in tumor cells, and a stronger and more diffuse Jagged-2 staining. In metastatic melanomas, ligands show predominantly plasma membrane staining, and Jagged-2 immunoreactivity appears decreased in the number of positive tumor cells and intensity in comparison with primary melanomas.

both the in situ and invasive component. However, in metastatic melanomas, Jagged-2 immunoreactivity appeared decreased, for number of positive cells and stain intensity, compared to primary melanomas. In contrast, no clear difference between primary and metastatic melanomas was observed for Notch-1, Notch-2, Jagged-1, and Delta-like 1.

Finally, when we examined the level of expression of Notch-1 and Notch-2 and their ligands in invasive melanomas, we found no association between protein levels and patients' clinicopathological and histopathological parameters or disease outcome.

\section{Discussion}

We have conducted an extensive investigation of the expression of Notch receptors and their ligands in benign and malignant cutaneous melanocytic lesions. In this report, we provide evidence that Notch-1 and Notch-2, as well as their ligands Jagged1, Jagged-2, and Delta-like 1, are significantly upregulated in 'dysplastic nevi' and melanomas, compared to common melanocytic nevi. Our results indicate that the activation of the Notch signaling may represent an early event in melanocytic tumor progression and support the hypothesis that the Notch pathway may have a role in inducing and progression of neoplastic transformation.

Despite accumulating information concerning the Notch signaling in cell fate determination and cell differentiation, the molecular mechanisms through which the Notch signaling participates in cancerogenesis is complex and still far from fully understood. The Notch proteins appear to have dual opposing properties resulting in a net oncogenic or oncosuppressive function depending of the cellular and tumoral context. In addition, a recent report has suggested that Notch-1 may have a dose-dependent 
function in cancer, with low levels of Notch-1 protecting cervical cancer cells from proapoptotic signals, and thus being beneficial to cancer cells, and higher levels of Notch-1 activity being detrimental, driving neoplastic cells into G1 arrest. ${ }^{2,29,30}$

A number of studies have focused on the possible involvement of the Notch signals in the deregulation of components of the cell cycle machinery. ${ }^{31}$ Since nontransforming Notch $^{\text {ic }}$ mutant proteins fail to activate cyclin D1 transcription, it has been suggested that the oncogenic mechanism driven by Notch $^{\text {ic }}$ involves, in part, the deregulation of the cell cycle through overexpression of cyclin D1 and activation of CDK2. ${ }^{31}$ Conversely, in small-cell lung cancer cells, the Notch signaling has been associated with growth inhibition through G1 arrest in the G1 phase as well as specific activation of p21 waf/cip1 and p27 $7^{\text {kip132 }}$ Similarly, a significant growth inhibition related to G0/G1 cell cycle arrest was demonstrated in hepatocellular carcinoma, where Notch-1 signaling was found to downregulate the expression of cyclin A1, cyclin D1, cyclin E, and CDK2, upregulate $\mathrm{p} 21^{\text {waf/cip } 1}$ expression and induce p53 expression, and thus apoptosis. ${ }^{33}$

Concerning Notch-1 involvement in apoptotic processes, however, there are controversial results depending on cell type and tissue context. While in liver carcinoma Notch-1 signaling results in apoptosis induction, ${ }^{33}$ in lymphoma or myeloma cells ${ }^{12,34}$ and in cholangiocarcinoma cells, ${ }^{15}$ Notch-1 activation appears to confer an apoptosis-resistant phenotype to neoplastic cells. Interestingly, in the context of cholangiocyte carcinogenesis, it has been shown that inducible nitric oxide synthase (iNOS) induced Notch-1 expression, via NO generation. Moreover, iNOS facilitated Notch signaling by facilitating nuclear translocation of its intracellular domain and expression of Hes1, a Notch transcriptional target. ${ }^{15}$ The relationship between these two proteins is intriguing in view of our previous observations that iNOS mRNA and protein were constantly absent in melanocytic nevi, but frequently expressed in melanomas, with a positive correlation between enzyme levels and tumor progression. ${ }^{35}$ Thus, iNOS may play a role in malignant transformation of melanocytes not only by inducing neovascularization, DNA damage, inhibition of DNA repair, and upregulation of COX2 but also by inducing antiapoptotic signals through Notch-1 expression. ${ }^{15}$

Effects resulting from physical interaction between ligands and respective Notch receptors are multiple and remain largely unexplored in their functional significance. Previous studies have demonstrated that soluble, nontransmembrane, form of Jagged-1 may act as agonist, as well as antagonist, of Notch signaling, possibly interfering with fulllength ligand interaction with Notch receptors. ${ }^{36,37}$ Of interest, thrombin, the key enzyme of the coagulation cascade, is able to cleave Jagged-1, producing the soluble form of the ligand (I Prudovs- ky, personal communication). Several reports indicate that thrombin participates in melanoma growth and metastasis. ${ }^{38}$ We have recently reported that thrombin receptor PAR-1 is unregulated in the initial phases of melanocytic tumor transformation and progression ${ }^{39}$ and it has been shown that in cutaneous melanoma, loss of expression of the transcription factor activator protein-2 $\alpha$ (AP-2), correlates with overexpression of PAR-1 and the acquisition of a malignant phenotype..$^{40}$ Thus, it may be proposed that thrombin participates in melanoma progression by different signaling pathways, including PAR-1 and Jagged-1 cleavage.

In conclusion, Notch-1 and Notch-2 displayed a significant increase in their expression levels, from benign to malignant cutaneous melanocytic lesions. In addition, present data showed that all Notch ligands were also upregulated, thus suggesting that overactivation of the Notch pathway occurs in melanoma. Experiments aimed at characterizing the effect of gain or loss of Notch function in melanoma cells will be necessary to uncover the type of involvement of Notch-mediated intracellular signaling in melanoma development.

\section{Acknowledgements}

This study was financially supported by grant from Ministero dell'Istruzione, Università e Ricerca (MIUR) (PRIN 2004) \#2004063945 to GC and MIUR (PRIN 2004) \#2004062950 to MS.

\section{References}

1 Artavanis-Tsakonas S, Rand MD, Lake RJ. Notch signaling: cell fate control and signal integration in development. Science 1999;284:770-776.

2 Jeffries S, Capobianco AJ. Neoplastic transformation by Notch requires nuclear localization. Mol Cell Biol 2000;20:3928-3941.

3 Allman D, Punt JA, Izon DJ, et al. An invitation to T and more: notch signaling in lymphopoiesis. Cell 2002; 109(Suppl):S1-S11.

4 Robbins J, Blondel BJ, Gallahan D, et al. Mouse mammary tumor gene int-3: a member of the notch gene family transforms mammary epithelial cells. J Virol 1992;66:2594-2599.

5 Jhappan C, Gallahan D, Stahle C, et al. Expression of an activated Notch-related int-3 transgene interferes with cell differentiation and induces neoplastic transformation in mammary and salivary glands. Genes Dev 1992; 6:345-355.

6 Lardelli M, Williams R, Lendahl U. Notch-related genes in animal development. Int J Dev Biol 1995;39: 769-780.

7 Gray GE, Mann RS, Mitsiadis E, et al. Human ligands of the Notch receptor. Am J Pathol 1999;154:785-794.

8 Shutter JR, Scully S, Fan W, et al. Dll4, a novel Notch ligand expressed in arterial endothelium. Genes Dev 2000;14:1313-1318. 
9 Schroeter EH, Kisslinger JA, Kopan R. Notch-1 signalling requires ligand-induced proteolytic release of intracellular domain. Nature 1998;393:382-386.

10 Levitan D, Lee J, Song L, et al. PS1 N- and C-terminal fragments form a complex that functions in APP processing and Notch signaling. Proc Natl Acad Sci USA 2001;98:12186-12190.

11 Zagouras P, Stifani S, Blaumueller CM, et al. Alterations in Notch signaling in neoplastic lesions of the human cervix. Proc Natl Acad Sci USA 1995;92: 6414-6418.

12 Jundt F, Anagnostopoulos I, Forster R, et al. Activated Notch1 signaling promotes tumor cell proliferation and survival in Hodgkin and anaplastic large cell lymphoma. Blood 2002;99:3398-3403.

13 Parr C, Watkins G, Jiang WG. The possible correlation of Notch-1 and Notch-2 with clinical outcome and tumour clinicopathological parameters in human breast cancer. Int J Mol Med 2004;14:779-786.

14 Purow BW, Haque RM, Noel MW, et al. Expression of Notch-1 and its ligands, Delta-like-1 and Jagged-1, is critical for glioma cell survival and proliferation. Cancer Res 2005;65:2353-2363.

15 Ishimura N, Bronk SF, Gores GJ. Inducible nitric oxide synthase up-regulates Notch-1 in mouse cholangiocytes: implications for carcinogenesis. Gastroenterology 2005;128:1354-1368.

16 Santagata S, Demichelis F, Riva A, et al. Jagged 1 expression is associated with prostate cancer metastasis and recurrence. Cancer Res 2004;64:6854-6857.

17 Weng AP, Aster JC. Multiple niches for Notch in cancer: context is everything. Curr Opin Genet Dev 2004;14:48-54.

18 Rangarajan A, Talora C, Okuyama R, et al. Notch signaling is a direct determinant of keratinocyte growth arrest and entry into differentiation. EMBO J 2001;20:3427-3436.

19 Nicolas M, Wolfer A, Raj K, et al. Notch1 functions as a tumor suppressor in mouse skin. Nat Genet 2003;33:416-421.

20 Sriuranpong V, Borges MW, Ravi RK, et al. Notch signaling induces cell cycle arrest in small cell lung cancer cells. Cancer Res 2001;61:3200-3205.

21 Thelu J, Rossio P, Favier B. Notch signalling is linked to epidermal cell differentiation level in basal cell carcinoma, psoriasis and wound healing. BMC Dermatol 2002;2:7.

22 Talora C, Sgroi DC, Crum CP, et al. Specific downmodulation of Notch1 signaling in cervical cancer cells is required for sustained HPV-E6/E7 expression and late steps of malignant transformation. Genes Dev 2002;16:2252-2263.

23 Talora C, Cialfi S, Segatto O, et al. Constitutively active Notch1 induces growth arrest of HPV-positive cervical cancer cells via separate signaling pathways. Exp Cell Res 2005;305:343-354.

24 Seykora JT, Jih D, Elenitsas R, et al. Gene expression profiling of melanocytic lesions. Am J Dermatopathol 2003;25:6-11.
25 Hoek K, Rimm DL, Williams KR, et al. Expression profiling reveals novel pathways in the transformation of melanocytes to melanomas. Cancer Res 2004;64: 5270-5282.

26 Gutgemann A, Golob M, Muller S, et al. Isolation of invasion-associated cDNAs in melanoma. Arch Dermatol Res 2001;293:283-290.

27 World Health Organization Classification of Tumours. Tumours of the Skin. IARC Press: Lyon, 2005 (in press).

28 Baldi A, De Falco M, De Luca L, et al. Characterization of tissue specific expression of Notch-1 in human tissues. Biol Cell 2004;96:303-311.

29 Lathion S, Schaper J, Beard P, et al. Notch1 can contribute to viral-induced transformation of primary human keratinocytes. Cancer Res 2003;63: 8687-8694.

30 Weijzen S, Zlobin A, Braid M, et al. HPV16 E6 and E7 oncoproteins regulate Notch-1 expression and cooperate to induce transformation. J Cell Physiol 2003;194: 356-362.

31 Ronchini C, Capobianco AJ. Induction of cyclin D1 transcription and CDK2 activity by Notch(ic): implication for cell cycle disruption in transformation by Notch(ic). Mol Cell Biol 2001;21:5925-5934.

32 Sriuranpong V, Borges MW, Ravi RK, et al. Notch signaling induces cell cycle arrest in small cell lung cancer cells. Cancer Res 2001;61:3200-3205.

33 Qi R, An H, Yu Y, et al. Notch1 signaling inhibits growth of human hepatocellular carcinoma through induction of cell cycle arrest and apoptosis. Cancer Res 2003;63:8323-8329.

34 Jundt F, Probsting KS, Anagnostopoulos I, et al. Jagged 1-induced Notch signaling drives proliferation of multiple myeloma cells. Blood 2004;103:3511-3515.

35 Massi D, Franchi A, Sardi I, et al. Inducible nitric oxide synthase expression in benign and malignant cutaneous melanocytic lesions. J Pathol 2001;194: 194-200.

36 Sun X, Artavanis-Tsakonas S. Secreted forms of DELTA and SERRATE define antagonists of Notch signaling in Drosophila. Development 1997;124:3439-3448.

37 Small D, Kovalenko D, Soldi R, et al. Notch activation suppresses fibroblast growth factor-dependent cellular transformation. J Biol Chem 2003;278:1640516413.

38 Shi X, Gangadharan B, Brass LF, et al. Proteaseactivated receptors (PAR1 and PAR2) contribute to tumor cell motility and metastasis. Mol Cancer Res 2004;2:395-402.

39 Massi D, Naldini A, Ardinghi C, et al. Expression of protease-activated receptor-1 and -2 in melanocytic nevi and human cutaneous melanoma. Hum Pathol 2005;36:676-685.

40 Tellez C, McCarty M, Ruiz M, et al. Loss of activator protein- $2 \alpha$ results in overexpression of protease-activated receptor-1 and correlates with the malignant phenotype of human melanoma. J Biol Chem 2003; 278:46632-46642. 